Latest results from the SEMATECH Berkeley extreme ultraviolet microfield exposure tool

Patrick P. Naulleau, Christopher N. Anderson, Jerrin Chiu, Kim Dean, Paul Denham, Simi George, Kenneth A. Goldberg, Brian Hoef, Gideon Jones, Chawon Koh, Bruno La Fontaine, Andy Ma, Warren Montgomery, Dimitra Niakoula, Joo-On Park, Tom Wallow, Stefan Wurm

\author{
Center for X-ray Optics, Lawrence Berkeley National Laboratory \\ University of California, Berkeley \\ SEMATECH, Albany, NY \\ Advanced Micro Devices, Sunnyvale, CA \\ Intel Corporation, Santa Clara, CA \\ Samsung Electronics, Korea
}

Completed January 2008

This work was supported by the Director, Office of Science, Office of Basic Energy Sciences, of the U.S. Department of Energy under Contract No. DE-AC02-05CH11231 


\title{
Latest results from the SEMATECH Berkeley extreme ultraviolet microfield exposure tool
}

\author{
Patrick P. Naulleau, ${ }^{1}$ Christopher N. Anderson, ${ }^{2}$ Jerrin Chiu, ${ }^{\prime}$ Kim Dean,${ }^{3}$ Paul Denham, \\ Simi George, ${ }^{1}$ Kenneth A. Goldberg, ${ }^{1}$ Brian Hoef, ${ }^{1}$ Gideon Jones, ${ }^{1}$ Chawon Koh, ${ }^{3}$ \\ Bruno La Fontaine, ${ }^{4}$ Andy $\mathrm{Ma},{ }^{5}$ Warren Montgomery, ${ }^{3}$ Dimitra Niakoula, ${ }^{1}$ Joo-on Park, ${ }^{6}$ \\ Tom Wallow, ${ }^{4}$ Stefan Wurm ${ }^{3}$ \\ ${ }^{\prime}$ Center for X-Ray Optics, Lawrence Berkeley National Laboratory, Berkeley, CA 94720 \\ ${ }^{2}$ Applied Sci. \& Technol. Graduate Group, University of California, Berkeley, CA 94720 \\ ${ }^{3}$ SEMATECH, Albany, NY 12203 \\ ${ }^{4}$ Advanced Micro Devices, Sunnyvale, CA 94088 \\ ${ }^{5}$ Intel Corporation, Santa Clara, CA 95052 \\ ${ }^{6}$ Samsung Electronics, Korea
}

\begin{abstract}
Microfield exposure tools (METs) continue to play a dominant role in the development of extreme ultraviolet (EUV) resists. One of these tools is the 0.3 numerical aperture SEMATECH Berkeley MET operating as a resist and mask test center. Here we present an update on the tool summarizing some of the latest test and characterization results. We provide an update on the long-term aberration stability of the tool and present line-space imaging in chemically amplified photoresist down to the 20-nm half-pitch level. Although resist development has shown substantial progress in the area of resolution, line-edge-roughness (LER) remains a significant concern. Here we present a summary of recent LER performance results and consider the effect of mask contributors to the LER observed from the SEMATECH Berkeley microfield 100 .
\end{abstract}

\section{Introduction}

Despite the recent availability of full field extreme ultraviolet (EUV) alpha tools $[1,2]$, microfield exposure systems [3-5] continue to play a crucial role in the development of EUV lithography. In general, such tools provide enhanced imaging capabilities over their significantly more complex full-field scanning counterparts. One reason for the enhanced 
imaging capabilities provided by microfield systems is the larger numerical apertures (NAs): 0.3 for current microfield systems as compared to 0.25 for full field systems. For the SEMATECH Berkeley microfield exposure tool (MET) [3], the imaging capabilities are further enhanced by the lossless programmable pupil-fill illuminator [6] enabling low $k_{1}$ imaging. These superior imaging capabilities have become increasingly important as the EUV high volume manufacturing entry node is expected to be delayed to the 22-nm half pitch node. For example, assuming a $k_{1}$ factor of 0.45 , at $0.25-\mathrm{NA}$ the resolution limit would be $24 \mathrm{~nm}$, whereas at $0.3-\mathrm{NA}$ the resolution limit is $20 \mathrm{~nm}$.

In this manuscript we report on recent resist characterization results from the SEMATECH Berkeley MET tool demonstrating 20-nm resolution in chemically amplified resist. Moreover, we present aberration characterization results demonstrating the long-term stability of the projection optics box. Although recent resist characterization has shown very promising results from the perspective of resolution; line-edge roughness (LER) remains a significant concern. Here we summarize modeling results evaluating the contribution of mask effects on the measured LER.

\section{Projection optics stability}

The primary uses of the SEMATECH Berkeley MET (BMET) are in the evaluation of resists and mask architectures. Tool stability is crucial to these uses since it allows processes and/or masks to be compared over long time periods. Another active area of research using the BMET is in the development of fundamental resist characterization metrics such as deprotection blur (intrinsic resolution) metrics [7-11]. Being based on the comparison of acrial image modeling results to actual resist exposures to extract the resist 
metrics, these methods rely sensitively on accurate knowledge of the projection optics aberrations, again making long term stability key.

To assess the long term stability of the BMET we have lithographically characterized the field dependent astigmatism over a two and a half year period. Complete field measurements were performed in 2005 and 2007 while in 2006 we performed only a partial field measurement. The aberration values reported in Fig. 1 are rms magnitudes over the annular MET pupil in $\mathrm{nm}$. The relative locations in the tables correspond to the relative measurement locations in the $600 \times 200 \mu \mathrm{m}$ field. The horizontal and vertical measurement field point separations are approximately $200 \mu \mathrm{m}$ and $60 \mu \mathrm{m}$, respectively. Given the $0.1-\mathrm{nm}$ rms uncertainty in the measurements [11], the results in Fig. 1 show the optic alignment to be very stable over time.

\section{Resist characterization results}

Significant improvement in resist performance has been achieved over the past year. Figure 2 shows printing results from a resist with a dose to size on $30-n m$ equal linespace features of approximately $12 \mathrm{~mJ} / \mathrm{cm}^{2}$. The resist thickness is $50 \mathrm{~nm}$ and the illumination conditions were $45^{\circ}$ rotated dipole with a pole radius of 0.175 and an offset of 0.53 . This material show's well resolved lines down to $26-\mathrm{nm}$ half pitch and modulation down to $22 \mathrm{~nm}$. In Fig. 3 we show two materials with resolution capabilities of $20 \mathrm{~nm}$. Pattern collapse is seen to be the dominant effect in the resolution limit, suggesting that the intrinsic resolution capabilities are even better. The sensitivities of resists $A$ and B in Fig. 3 are $12.7 \mathrm{~mJ} / \mathrm{cm}^{2}$ and $15.2 \mathrm{~mJ} / \mathrm{cm}^{2}$, respectively. Again the resist thickness is $50 \mathrm{~nm}$ and the illumination conditions were $45^{\circ}$ rotated dipole but this time with a pole radius of 0.1 and an offset of 0.57 . A direct comparison of the modeled aerial- 
image contrast for the two different rotated dipole settings from Figs. 2 and 3 is shown in Fig. 4. Although the latter illumination setting does not change the resolution limit in terms of the roll off point in the contrast transfer function, it does provide a general contrast enhancement of approximately $15 \%$ absolute.

Although significant improvements have been made in the areas of resolution and sensitivity for EUV resists, line edge roughness (LER) remains a significant challenge. In order to address this issue a variety of process-based approaches such as specialized rinse agents [12-14] and resist underlayers [15] are being considered. In Figs. 5 and 6 we show an example of the effectiveness of underlayers in the control of LER. The resist film thickness is $50 \mathrm{~nm}$ and the underlayer thickness is $40 \mathrm{~nm}$. The illumination conditions were annular $0.35<\sigma<0.55$. Figure 5 shows a direct comparison of through-focus imaging at a series of different feature sizes for the same resist exposed with two different underlayers. Figure 6 shows plots of the LER corresponding to the imaging results in Fig. 5. The results show a change in LER at best focus of approximately 15\% and demonstrate the strong impact of underlayers.

\section{Mask contributors to LER}

The LER challenge is well summarized by the scatter plot in Fig. 7 showing LER versus sensitivity from a wide variety of resists tested on the BMET over the past four years. A lower LER limit is evident suggesting that the manufacturing LER specifications cannot be met with current resists no matter how slow they are made. This raises the interesting question of what is actually dominating the LER limit since it is evidently not sensitivity. One important potential contributor is the mask [16-18]. The mask can contribute to resist LER in two ways: pattern LER on the mask itself as well as reflector or phase roughness. 
In this section we summarize the predicted mask contribution to LER in the BMET [19] and consider the implications on the LER limit observed in Fig. 7.

The BMET mask effects on LER are predicted using aerial-image modeling. Scanning electron micrographs of the mask are used to determine the mask LER and synthesize a simulation mask with the same LER properties. Atomic force microscope characterization of the mask is further used to determine the surface roughness and resulting phase roughness. This phase roughness is then added to the clear regions of the simulation mask. For more details on this process, see Ref. [19]. Considering the baseline annular illumination from the BMET, Fig. 8 shows the modeled image-plane LER contributions from the mask. At best focus, the mask pattern LER is seen to be the dominant term, whereas as we move away from focus the multilayer phase roughness begins to dominate. Because the two effects are uncorrelated on the mask, the combined effect is essentially the quadrature sum of the two individual effects. At best focus, the combined effect yields approximately $1.4 \mathrm{~nm}$ of LER, whereas $100-\mathrm{nm}$ out of focus, the effect jumps to $2 \mathrm{~nm}$ and even larger depending on the direction of the defocus. These results show that good focus control is essential to minimizing the mask contributions to LER and that even at best focus the mask effects are not negligible. Figure 9 shows the same LER plot from Fig. 7 but corrected for the modeled mask contribution where we have assumed the best focus condition. The mask contribution is assumed to be uncorrelated from the remainder of the LER contributors and is thus removed by quadrature subtraction. Due to this quadrature subtraction, the impact is less dramatic than one might initially suspect based simply on the LER values in Fig. 8.

\section{Summary}


EUV resist resolution capabilities down to the $20-\mathrm{nm}$ half pitch level have been demonstrated as reasonable sensitivities. The use of underlayers has been shown to have strong impact on resist LER characteristics. Despite these gains, LER remains a significant challenge. Aerial image modeling has been used to show that mask effects can no longer be assumed to be negligible in printed LER. Nevertheless, even after compensating for mask effects, measured LER still displays a significant lower limit.

\section{Acknowledgements}

The authors are greatly indebted to Kevin Bradley, Rene Delano, Jeff Gamsby, Eric Gullikson, Bob Gunion, Gideon Jones, Ron Oort, Ron Tackaberry, and Farhad Salmassi for expert engineering, technical, and fabrication support. The authors are also grateful to Koki Tamura, Chris Rosenthal, and Dave White of TOK, Shinji Tarutani of Fujifilm, and Jim Thackeray and Katherine Spear of Rohm and Haas for resist support. This work was supported by SEMATECH and carried out at Lawrence Berkeley National Laboratory's Advanced Light Source, which is supported by the DOE, Office of Science, Basic Energy Sciences.

\section{References}

1. H. Meiling, et al., "First performance results of the ASML alpha demo tool," Proc. SPIE 6151, 615108 (2006).

2. M. Miura, K. Murakami, K. Suzuki, Y. Kohama, Y. Ohkubo, T. Asami, "Nikon EUVL development progress summary," Proc. SPIE 6151, 615105 (2006).

3. P. Naulleau, et al., "Status of EUV micro-exposure capabilities at the ALS using the 0.3-NA MET optic," Proc. SPIE 5374, 881-891 (2004). 
4. A. Brunton, et al., "High-resolution EUV imaging tools for resist exposure and aerial image monitoring," Proc. SPIE 5751, 78-89 (2005).

5. H. Oizumi, Y. Tanaka, I. Nishiyama, H. Kondo, K. Murakami, "Lithographic performance of high-numerical-aperture $(N A=0.3)$ EUV small-field exposure tool (HDNA)," Proc. SPIE 5751, 102-109 (2005).

6. P. Naulleau, K. Goldberg, P. Batson, J. Bokor, P. Denham, and S. Rekawa, "A Fouriersynthesis custom-coherence illuminator for EUV microfield lithography," Appl. Opt. 42, $820-826(2003)$

7.P. Naulleau and C. Anderson "Lithographic metrics for the determination of intrinsic resolution limits in EUV resists," Proc. SPIE 6517, 65372N (2007).

8. C. Anderson and P. Naulleau, "Sensitivity study of two high-throughput resolution metrics for photoresists," Appl. Opt. 47, 56-63 (2008).

9. Y. Tanaka, Y. Kikuchi, D. Goo, and I. Nishiyama, "Fidelity of rectangular patterns printed with 0.3-NA MET optics," Proc. SPIE 6517, 65172L (2007).

10. C. Anderson and P. Naulleau, "A high-throughput contact-hole resolution metric for photoresists: full-process sensitivitystudy," Proc. SPIE 6923, to be published (2008).

11. G. M. Gallatin, P. Naulleau, D. Niakoula, R. Brainard, E. Hassanein, R. Matyi, J. Thackeray, K. Spear, K. Dean, "Resolution, LER and sensitivity limitations in photoresists," Proc. SPIE 6921, to be published (2008).

11. P. Naulleau, J. Cain, K. Goldberg, "Lithographic characterization of the field dependent astigmatism and alignment stability of a 0.3 numerical aperture EUV microfield optic," J. Vac. Sci. \& Technol. B 23, 2003-2006 (2005). 
12. P. Zhang, M. B. Rao, M. Jaramillo Jr., B. Horvath, B. Ross, T. Paxton, T. Davis, P. Cook, D. Witko, "Pattern Collapse and Line Width Roughness Reduction by Surface Conditioner Solutions for $248 \mathrm{~nm}$ Lithography," Proc. SPIE 5753, 252, (2005).

13. P. Wong, W. Gehoel, S. Sinkwitz, P. Zhang, M. Jaramillo Jr., M. B. Rao, B. Horvath, B. Ross, S. Cassel, "Linewidth Roughness Reduction at the $55 \mathrm{~nm}$ Node Through Combination of Classical Process Optimization and Application of Surface Conditioner Solutions," Proc. SPIE 6153, 61533V, (2006).

14. T. Wallow, R. Kim, B. La Fontaine, P. Naulleau, C. Anderson, R. Sandberg, "Progress in EUV Photoresist Technology," Proc SPIE 6533, 653317 (2007).

15. A. Ma, J. Park, K. Dean, S. Wurm, P. Naulleau, "Advanced extreme ultraviolet resist testing using the SEMATECH Berkeley 0.3-NA microfield exposure tool," Proc SPIE 6921, to be published (2008).

16. P. Naulleau and G. Gallatin, "The line-edge roughness transfer function and its application to determining mask effects in EUV resist characterization," Appl. Opt. 42, $3390-3397(2003)$

17. V. Beaudry, T. Milster, "Effects of mask roughness and condenser scattering in EUVL systems," Proc. SPIE. 3676, 653-662 (1999).

18. P. Naulleau, "The relevance of mask-roughness-induced printed line-edge roughness in recent and future EUV lithography tests," Appl. Opt. 43, 4025-4032 (2004).

19. P. Naulleau, D. Niakoula, G. Zhang, "System-level line-edge roughness limits in extreme ultraviolet lithography," J. Vac. Sci. \& Technol. B, to be published (2008),

\section{List of Figures}


Fig. 1. Time dependence of lithographically measured astigmatism across the field. Reported results are rms magnitudes in nm. The locations in the table correspond to the physical locations of the measured site in the field.

Fig. 2. Printing results from a resist with a dose to size on 30-nm equal line-space features of approximately $12 \mathrm{~mJ} / \mathrm{cm}^{2}$. The resist thickness is $50 \mathrm{~nm}$ and the illumination conditions were $45^{\circ}$ rotated dipole with a pole radius of 0.175 and an offset of 0.53 .

Fig. 3. Two resists with resolution capabilities of $20 \mathrm{~nm}$. The sensitivities of resists $A$ and $B$ are $12.7 \mathrm{~mJ} / \mathrm{cm}^{2}$ and $15.2 \mathrm{~mJ} / \mathrm{cm}^{2}$, respectively. The resist thickness is 50 $\mathrm{nm}$ and the illumination conditions were $45^{\circ}$ rotated dipole but this time with a pole radius of 0.1 and an offset of 0.57 .

Fig. 4. Direct comparison of the modeled aerial-image contrast for the two different rotated dipole settings from Figs. 2 and 3, respectively.

Fig. 5. Direct comparison of through-focus imaging at a series of different feature sizes for the same resist exposed on top of two different underlayers, (a) and (b). respectively.

Fig. 6. Plots of the LER conresponding to the imaging results in Fig. 5.

Fig. 7. LER versus sensitivity from a wide variety of resists tested on the BMET over the past four years.

Fig. 8. Modeled image-plane LER contributions from the mask assuming annular illumination.

Fig. 9. LER from Fig. 7 corrected for the modeled mask contribution assuming best focus condition. Mask contribution is removed by quadrature subtraction. 


\section{$5 / 2005$}

$0^{\circ}$ astigmatism (rms nm)

\begin{tabular}{|c|c|c|}
\hline-0.5 & -0.4 & -0.2 \\
\hline-0.5 & -0.4 & 0.0 \\
\hline-0.5 & -0.5 & 0.0 \\
\hline
\end{tabular}

$45^{\circ}$ astigmatism (rms nm)

\begin{tabular}{|c|c|c|}
\hline 0.0 & -0.2 & -0.3 \\
\hline 0.0 & -0.1 & -0.2 \\
\hline 0.0 & -0.1 & -0.2 \\
\hline
\end{tabular}

Total magnitude (rms nm)

\begin{tabular}{|c|c|c|}
\hline 0.5 & 0.5 & 0.4 \\
\hline 0.5 & 0.4 & 0.2 \\
\hline 0.5 & 0.5 & 0.2 \\
\hline
\end{tabular}

$7 / 2006$

$0^{\circ}$ astigmatism (rms $\mathrm{nm}$ )

\begin{tabular}{|l|l|l|}
\hline & -0.3 & \\
\hline & -0.4 & \\
\hline & -0.3 & \\
\hline
\end{tabular}

$45^{\circ}$ astigmatism (rms nm)

\begin{tabular}{|c|c|c|}
\hline & -0.2 & \\
\hline & -0.2 & \\
\hline & 0.0 & \\
\hline
\end{tabular}

Total magnitude (rms nm)

\begin{tabular}{|l|l|l|}
\hline & 0.4 & \\
\hline & 0.4 & \\
\hline & 0.3 & \\
\hline
\end{tabular}

$10 / 2007$

$0^{\circ}$ astigmatism ( $\mathrm{ms} \mathrm{nm}$ )

\begin{tabular}{|c|c|c|}
\hline-0.5 & -0.5 & -0.2 \\
\hline-0.3 & -0.5 & -0.3 \\
\hline-0.1 & -0.2 & -0.1 \\
\hline
\end{tabular}

$45^{\circ}$ astigmatism (rms $\mathrm{nm}$ )

\begin{tabular}{|c|c|c|}
\hline-0.1 & 0.0 & 0.0 \\
\hline 0.0 & -0.1 & 0.0 \\
\hline-0.1 & -0.1 & -0.2 \\
\hline
\end{tabular}

Total magnitude ( $\mathrm{ms} \mathrm{nm}$ )

\begin{tabular}{|c|c|c|}
\hline 0.5 & 0.5 & 0.2 \\
\hline 0.3 & 0.5 & 0.3 \\
\hline 0.1 & 0.3 & 0.3 \\
\hline
\end{tabular}

Fig. 1. Time dependence of lithographically measured astigmatism across the field. Reported results are rms magnitudes in $\mathrm{nm}$. The locations in the table correspond to the physical locations of the measured site in the field. 


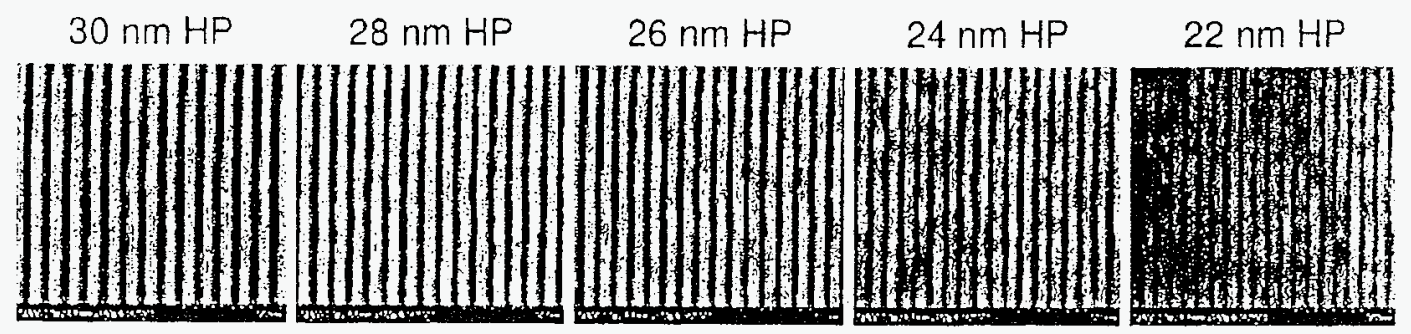

Fig. 2. Printing results from a resist with a dose to size on 30-nm equal line-space features of approximately $12 \mathrm{~mJ} / \mathrm{cm}^{2}$. The resist thickness is $50 \mathrm{~nm}$ and the illumination conditions were $45^{\circ}$ rotated dipole with a pole radius of 0.175 and an offset of 0.53 . 

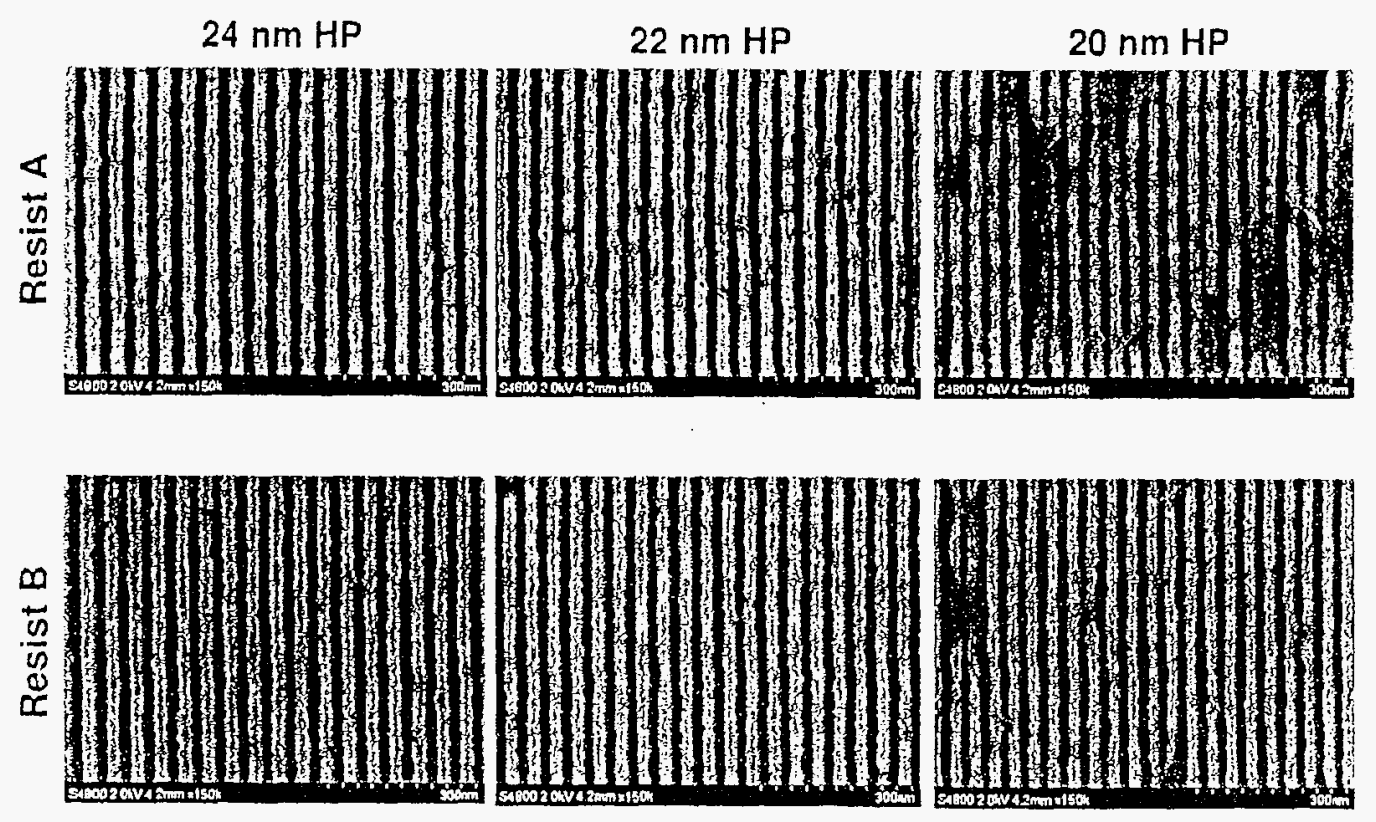

Fig. 3. Two resists with resolution capabilities of $20 \mathrm{~nm}$. The sensitivities of resists $A$ and $B$ are $12.7 \mathrm{~mJ} / \mathrm{cm}^{2}$ and $15.2 \mathrm{~mJ} / \mathrm{cm}^{2}$, respectively. The resist thickness is $50 \mathrm{~mm}$ and the illumination conditions were $45^{\circ}$ rotated dipole but this time with a pole radius of 0.1 and an offset of 0.57 


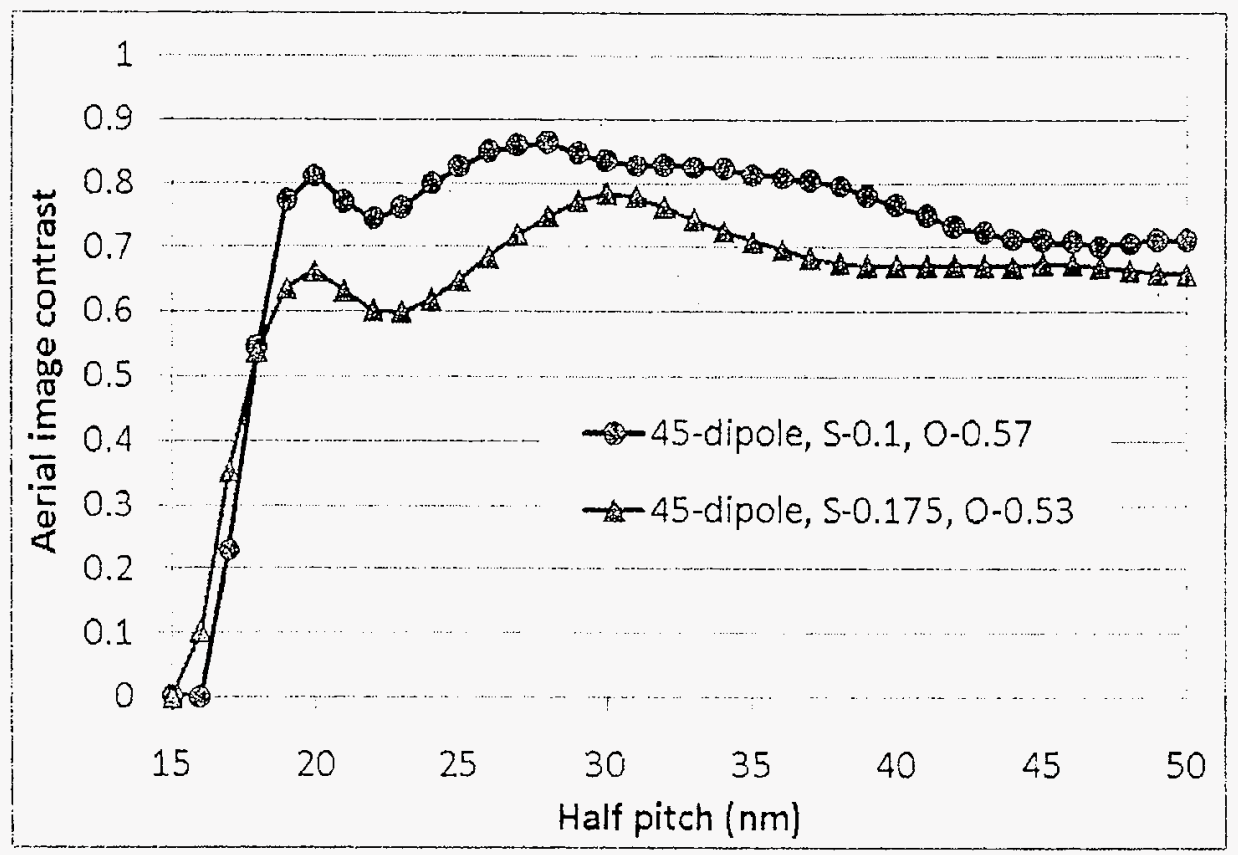

Fig. 4. Direct comparison of the modeled aerial-image contrast for the two different rotated dipole sertings from Figs. 2 and 3, respectively. 



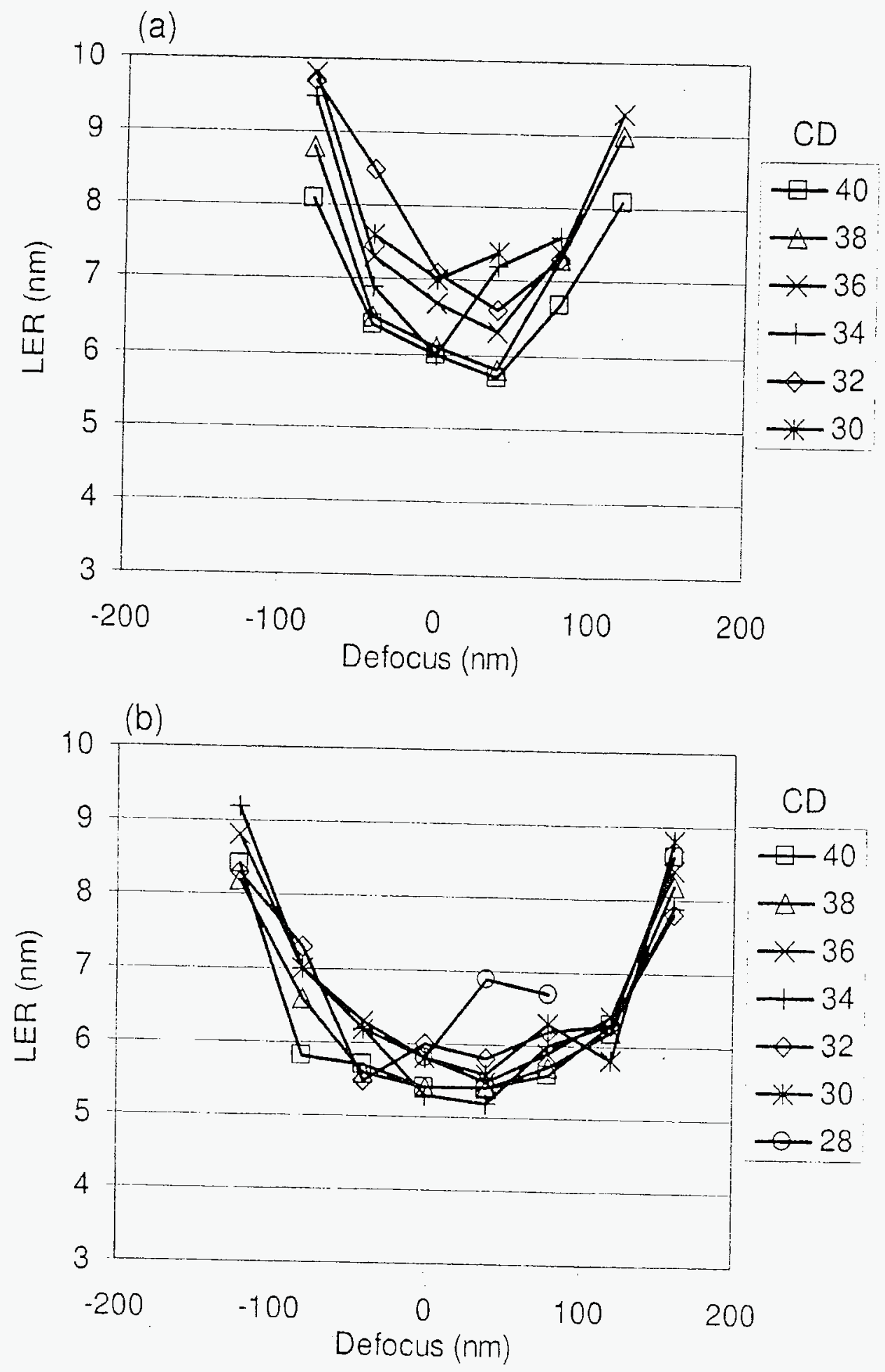


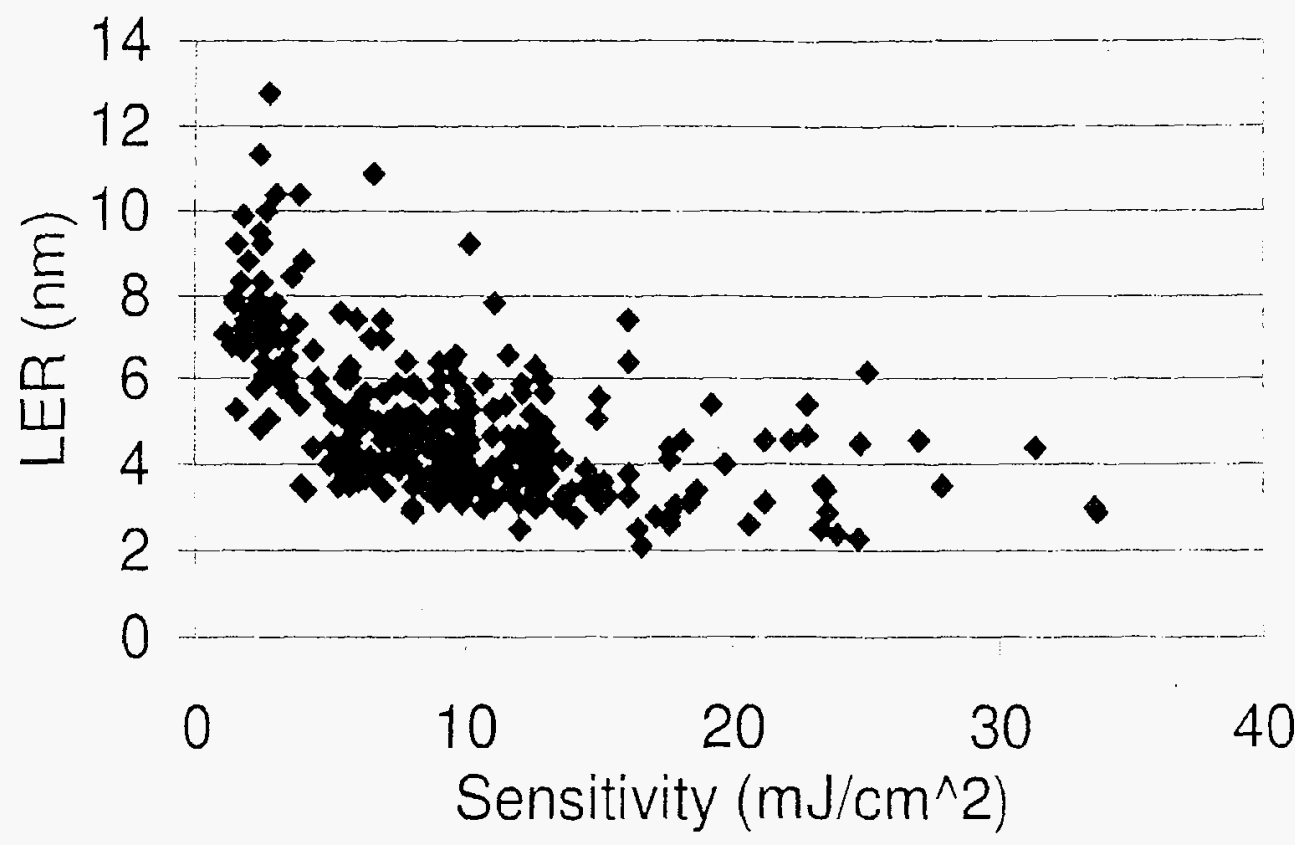

Fig. 7. LER versus sensitivity from a wide variety of resists tesied on the BMET over the past four years. 


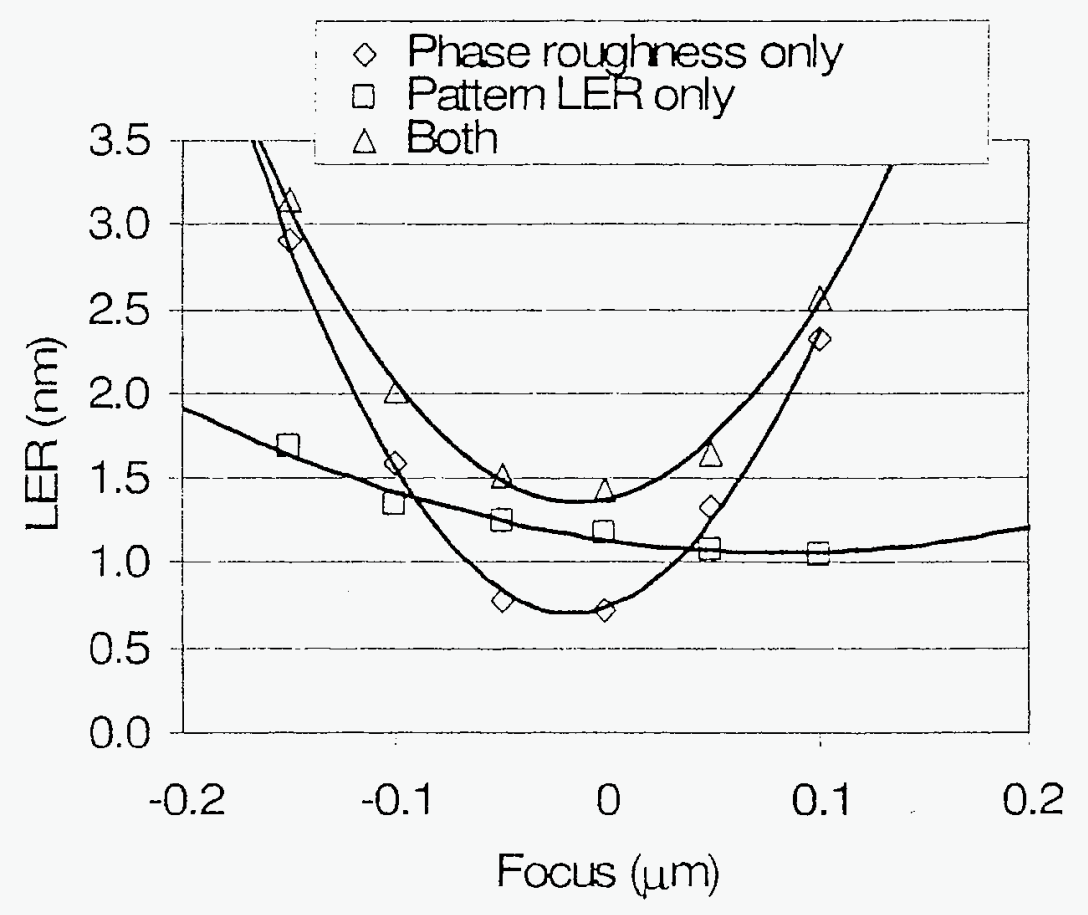

Fig. 8. Modeled image-plane LER contributions from the mask assuming annular illumination. 


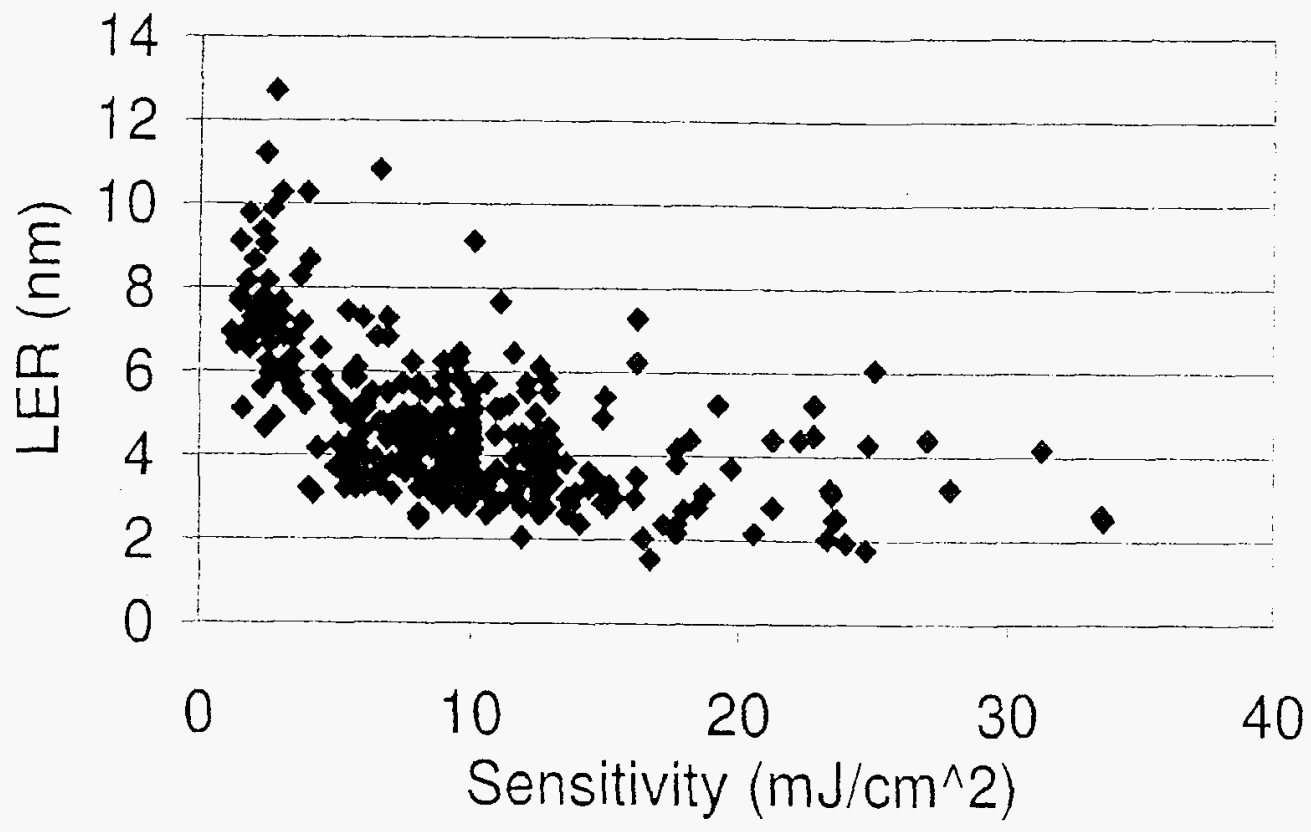

Fig. 9. LER from Fig. 7 corrected for the modeled mask contribution assuming best focus condition. Mask contribution is removed by quadrature subtraction. 
